\title{
Design, Simulation and Verification of a 7- DOF Joint Motion Simulation Platform
}

\author{
Ze CUI a,1, Saishuai HUANG ${ }^{\mathrm{a}}$, Zenghao CHEN ${ }^{\mathrm{a}}$, Hongxin $\mathrm{YANG}^{\mathrm{a}}$ and Danjie ZHU ${ }^{\mathrm{b}}$ \\ ${ }^{a}$ School of Mechatronic Engineering and Automation, Shanghai University, Shanghai, \\ China \\ ${ }^{b}$ Orthopedics Dept, Zhejiang Provincial People's Hospital, Zhejiang, China
}

\begin{abstract}
The human body has many joints, and joint injuries frequently occur in various sports. To explore the biomechanical state of ligaments or muscles in human joints before and after damage, and to help doctors judge the damage and repair of joints, this article proposes a seven-degree-of-freedom platform based on three rotations spherical parallel mechanism for simulating human joint motion. Taking the knee joint as an example, this article simplified its model, and performed kinematics simulation by ADAMS to verify the feasibility of this mechanism. And based on the TRIO motion controller, we established the physical testing system. The correctness is finally verified by experiment in kind, which proves the feasibility of the joint motion simulation platform. And in terms of accuracy, it also performances very well. For example, when it needs to rotate $30^{\circ}$ around the $\mathrm{Y}$-axis, its actual rotation angle is $29.6^{\circ}$, the error is less than $2 \%$, and its translation error is also within $3 \%$.
\end{abstract}

Keywords. seven degrees of freedom, joints, spherical parallel mechanism.

\section{Introduction}

To understand the function of the knee joint properly, we need to study the In-Situ force and the In-Vivo force, which need to add information in the range of ligament relaxation between full extension and deep flexion. In recent years, many studies have confirmed the role of joint in limiting tibial movement, however, due to the lack of appropriate experimental equipment to measure the total force in the ligament while the joint moving, this research has been hampered.

So far, many medical and engineering scholars have made outstanding contributions to this research. From 1996, Woo et al. [1] used a Universal Force/Robot System (UFS), which can provide a six-degree-of-freedom (DOF) manipulator when studying the knee of Anterior Cruciate Ligament (ACL). In-Situ force measurement used a multidimensional force sensor at the end of the manipulator. Atarod et al. [2-3] of the University of Calgary used a 6 DOF parallel mechanism when studying the movement of the knee joint of sheep and cooperated with a redundant DOF clamping device to fix the Femur ACL Tibia Complex (FATC) of sheep. The femur and tibia were fixed to conduct a sheep gait simulation experiment and measure the In-Situ force of the sheep knee ACL. Noble et al. [4] designed a universal robot based on rope drive and built a

${ }^{1}$ Corresponding Author: Ze Cui, School of Mechatronic Engineering and Automation, Shanghai University,99 Shangda Road, Shanghai, China; E-mail: Cuize0421@126.com 
musculoskeletal test system to design experiments to verify. Zens et al. [5] used a multidegree-of-freedom joint motion simulator in the study of the length of the anterolateral ligament during the passive motion of the knee joint. Nesbitt et al. [6] used a KUKA robot to produce the ideal movement of the knee joint while exploring the effects of robot flexibility and bone bending on the knee kinematics simulation. In 2017, the Department of trauma, Innsbruck Medical University, developed a new type of knee biomechanical test-bed for in vitro evaluation of the knee joint [7]. The platform allows the kinematics of the knee joint to be studied in all six degrees of freedom; in 2018, the University of Aalborg proposed a new method for noninvasive and accurate measurement of knee joint relaxation in four degrees of freedom [8-12]. The joint measuring instrument combines a parallel manipulator and a 6-DOF force/torque sensor to reconstruct the relaxation measurement of tibiofemoral position and direction.

In this paper, we propose a 7-DOF joint simulation platform based on three rotations (3-RRR) spherical parallel mechanism, so that can we find a more accurate method to evaluate the mechanical function of the human joint and internal tissue. And based on the TRIO motion controller, a physical testing system has been established. The purpose of this study is to provide help for the problems related to the residual relaxation of the human joint and adverse prognosis, meniscus injury, and graft failure.

\section{Design of Joint Motion Simulation Platform}

\subsection{Structure of Joint Motion Simulation Platform}

As we all know, the six degrees of freedom of space is composed of 3 rotations and three translations. Through research on existing institutions and positive thinking, in the end, a "3+3+1" degree of freedom joint motion simulation platform was designed (as shown in Figure 1).

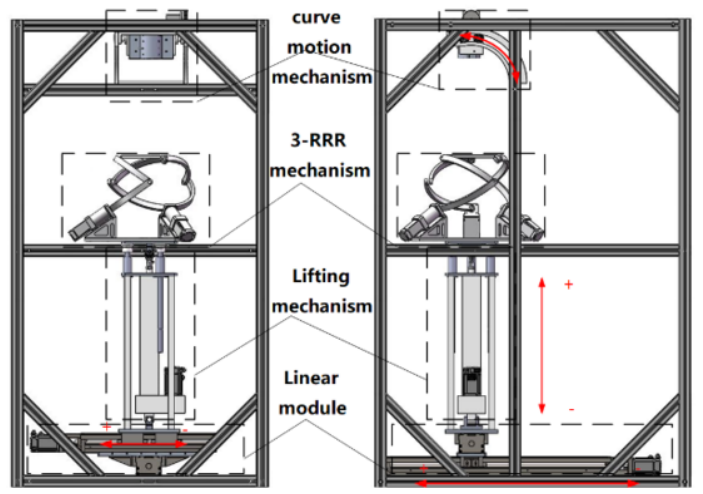

Figure 1. Structure diagram of the joint motion simulation platform

The mechanism is 1.08 meters long, 0.88 meters wide, and 1.6 meters high. It is mainly composed of an aluminum profile frame, an arc-shaped motion track, a 3-RRR mechanism, a lifting mechanism, and a two-dimensional linear module. The bottom twodimensional linear module can realize linear motion in the $\mathrm{X}$-axis and $\mathrm{Y}$-axis directions. An electric cylinder drives the lifting mechanism used to complete the linear movement in the Z-axis direction. The 3-RRR mechanism can realize three-degree-of-freedom motion in space. The arc-shaped motion mechanism can manually compensate for the 
pitch angle that the 3-RRR mechanism cannot reach. Through the cooperation of various mechanisms, six degrees of the freedom movement in space can be completed, thereby simulating human joints' movement. The movement space of the mechanisms is shown in Table 1.

Table 1. Movement Range of The Mechanism

\begin{tabular}{ccc}
\hline & Degree of freedom & Range of motion \\
\hline \multirow{2}{*}{ Rotation } & Pitch & $\pm 50^{\circ}$ \\
& Yaw & $-50^{\circ} \sim+140^{\circ}$ \\
\multirow{2}{*}{ Translation } & Roll & $\pm 50^{\circ}$ \\
& X & $-540 \mathrm{~mm} \sim 200 \mathrm{~mm}$ \\
& Y & $\pm 150 \mathrm{~mm}$ \\
& $\mathrm{Z}$ & $-20 \mathrm{~mm} \sim+140 \mathrm{~mm}$ \\
\hline
\end{tabular}

The advantage of this mechanism is that the 3-RRR mechanism is a spherical parallel mechanism, its motion is extremely flexible, and it can rotate three degrees of freedom around the center of motion, which is very similar to the rotation of human joints, so it is very suitable for simulating joints' movement. What's more, the research on the spherical parallel mechanism at home and abroad is relatively mature. There are many kinds of research on it at present [13-14] (for example, the Agile Wrist [15]), which can help us better control the movement required to complete the experiment.

\subsection{Control System of the Joint Motion Simulation Platform}

The control system of the joint motion simulation platform includes a laptop, a TRIO motion controller, 6 AC servo drivers and 6 AC servo motors, three of them are $400 \mathrm{~W}$ and the other three are $100 \mathrm{~W}$. Two $400 \mathrm{~W}$ drivers and motors are used to drive the motion of linear modules along $\mathrm{X}$-axis and $\mathrm{Y}$-axis, the left $400 \mathrm{~W}$ driver and motor are for the lifting mechanism, three $100 \mathrm{~W}$ drivers and motors are used to drive the 3-RRR mechanism. The control system diagram is shown in Figure 2. The laptop, TRIO controller and $\mathrm{AC}$ servo drivers are connected by network cable; they communicate through Ethernet protocol, the platform can be driven by the program written on the laptop.

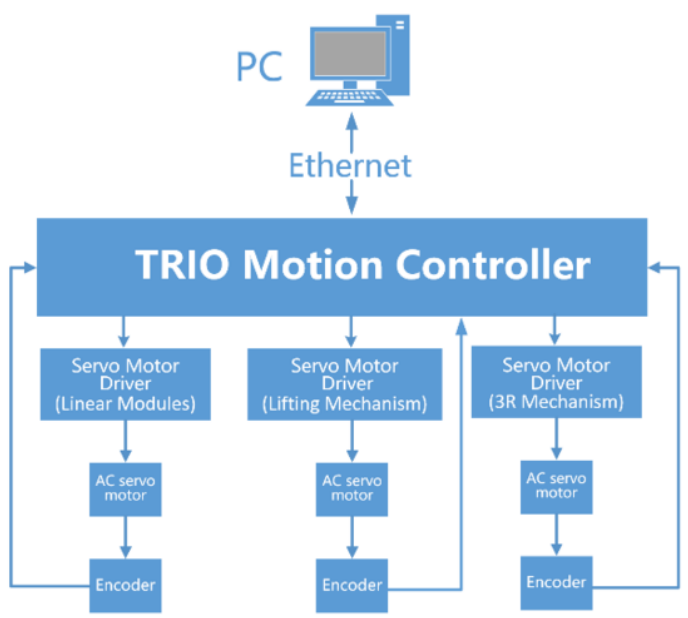

Figure 2. Control system block diagram 


\section{Adams Kinematics Simulation}

\subsection{Model Simplification of the Joint Motion Simulation Platform}

To facilitate the simulation in the ADAMS software, the model is equivalently simplified. As shown in Figure 3, the 3-RRR mechanism, the center of the upper round surface of the rod, is equivalent to the center of knee motion, and the center of the lower round surface is the center of rotation of the 3-RRR mechanism; other structures have also been simplified accordingly.

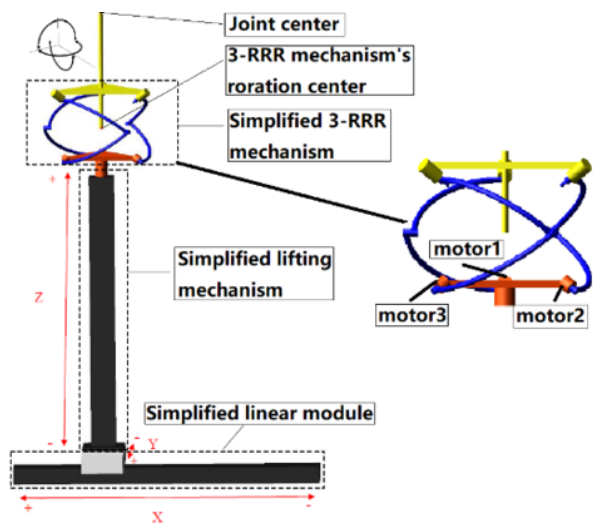

Figure 3. Simplified model of the joint motion simulation platform

\subsection{Motion Simulation Analysis}

According to the motion characteristics of each mechanism, the simplified model set the motion pair and drive and carried out some motion simulations. For example: Simulate the knee joint flexion movement of $30^{\circ}$, that is, the knee joint rotates $30^{\circ}$ around the $\mathrm{Y}$ axis to obtain the motion of each mechanism and the motion curve of the corresponding motion unit. In Figure 4, the pink dotted line represents the displacement in the Y-axis direction; the solid red line represents the displacement in the $\mathrm{X}$-axis direction; the red dotted line represents the displacement in the Z-axis direction; the black dotted line represents the rotation angle of the motor 1 in the 3-RRR mechanism; The solid green line and the light blue dotted line represent the rotation angles of the motor 2 and the motor 3 , respectively.

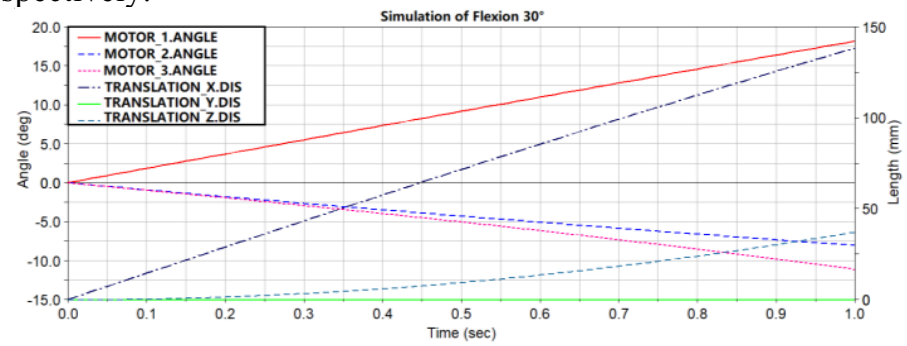

Figure 4. Simulate the knee joint flexion at $30^{\circ}$, the movement length/angle of each mechanism

\section{Experimental Verification}

To verify the feasibility of the joint motion simulation platform, a simple experiment has been done. 
In this experiment, a control system has been set up based on the TRIO motion controller. The hardware mainly consists of a motion controller (TRIO, MC 4N-ECAT, England), three 400W AC servo drivers (Panasonic, MBDLN25BE, Japan), three 100W AC servo drivers (Panasonic MADLN05BE, Japan), three 400W motors (Panasonic, MSMF042L1U2M, Japan), three 100W motors (Panasonic, MSMF012L1U2M, Japan). As shown in Figure 5, the platform performed well in the experiment.

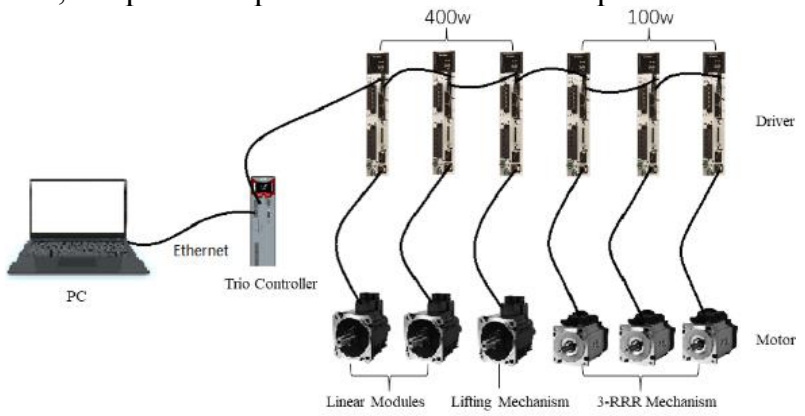

Figure 5. Control system connection diagram

The experiment process is as follows: a sheep's FATC was fixed to the platform. The platform drove sheep's FATC to rotate 30 degrees around Y-axis by a program. After the measurement, its actual rotation angle is $29.6^{\circ}$, the error is less than $2 \%$, and its translation error is also within 3\%. As shown in Figure 6, the platform performed well in the experiment.
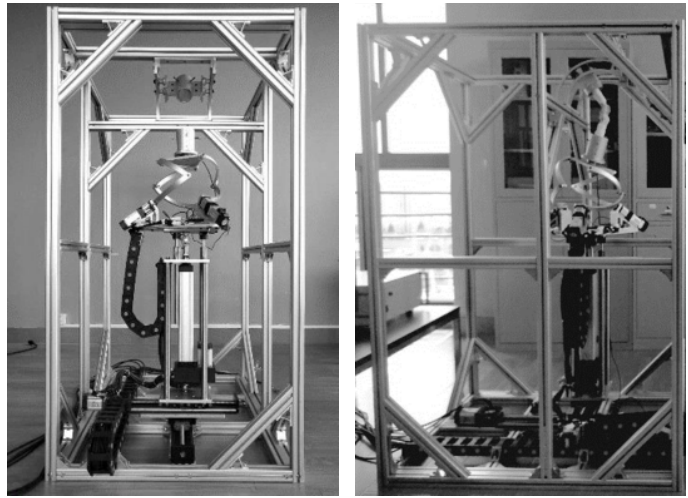

Figure 6. Experiment: sheep's FATC rotate $30^{\circ}$ around $\mathrm{Y}$-axis

\section{Conclusion}

This paper proposes a joint motion simulation platform based on a 3-RRR spherical parallel mechanism. Its motion center can be controlled at the joint's rotation center. It not only has a flexible space of seven degrees of freedom but also is easy to control. It is very suitable for studying human joint motion by simulating different movement states of human joints on the platform, which can perform In-Situ measurement of joints and various tissues in it. Once the mechanism is programmed, it can standardize the applied force's magnitude and direction to provide vectors (numbers and directions) to give less subjective joint measurements. And in terms of accuracy, it also performances very well, its rotation angle error is less than $2 \%$, and its translation error is also within $3 \%$. This 
article verifies the platform's feasibility and effectiveness through kinematic analysis, ADAMS motion simulation, and experiments, which ensure that their comprehensive knowledge, diagnosis, and treatment are highly relevant to clinicians.

At present, due to limited conditions, this platform can only simulate some simple low-speed movements of the joint.

In the future, it could be extended to include further movement patterns. We hope to use this platform to research joint dynamics, impact mechanics, muscle dynamics, etc. This will promote the development of the whole limb musculoskeletal discipline and better understand the human kinematics structure. Consequently, it would be possible to investigate emerging questions, particularly in joint trauma and rehabilitation optimization.

\section{References}

[1] Woo S L Y, Hollis J M, Adams D J, et al. Tensile properties of the human femur-anterior cruciate ligament-tibia complex: the effects of specimen age and orientation[J]. The American journal of sports medicine, 1991, 19(3): 217-225.

[2] Atarod M, Rosvold J M, Frank C B, et al. Functional activity of the anterior and posterior cruciate ligaments under in vivo gait and static physiological loads[J]. Annals of biomedical engineering, 2013, 41(10): 2067-2076.

[3] Atarod M, Rosvold J M, Frank C B, et al. A novel testing platform for assessing knee joint mechanics: a parallel robotic system combined with an instrumented spatial linkage[J]. Annals of biomedical engineering, 2014, 42(5): 1121-1132.

[4] Noble L D, Colbrunn R W, Lee D G, et al. Design and validation of a general purpose robotic testing system for musculoskeletal applications[J]. Journal of Biomechanical engineering, 2010, 132(2).

[5] Zens M, Niemeyer P, Ruhhammer J, et al. Length changes of the anterolateral ligament during passive knee motion: a human cadaveric study[J]. The American journal of sports medicine, 2015, 43(10): 2545 2552.

[6] Nesbitt R J, Bates N A, Karkhanis T D, et al. Impacts of Robotic Compliance and Bone Bending on Simulated in vivo Knee Kinematics[J]. American journal of biomedical engineering, 2016, 6(1): 12.

[7] Green J T, Hale R F, Hausselle J, et al.. A Reconfigurable Multiplanar in Vitro Simulator for Real-time Absolute Motion with External and Musculotendon Forces[J]. Journal of Biomechanical Engineering, 2017.

[8] Heinrichs C H, Knierzinger D, Stofferin H, et al. Validation of a novel biomechanical test bench for the knee joint with six degrees of freedom[J]. Biomedical Engineering/Biomedizinische Technik, 2018, 63(6): 709-717.

[9] Pedersen D, Vanheule V, Wirix-Speetjens R, et al. A novel non-invasive method for measuring knee joint laxity in four DOFdof: In vitro proof-of-concept and validation[J]. Journal of biomechanics, 2019, 82: $62-69$.

[10] Bates N A, Schilaty N D, Krych A J, et al. Influence of relative injury risk profiles on anterior cruciate ligament and medial collateral ligament strain during simulated landing leading to a noncontact injury event[J]. Clinical biomechanics, 2019, 69: 44-51.

[11] Schall F, Seitz A M, Hacker S, et al. Proof-of-concept of a novel knee joint simulator allowing rapid motions at physiological muscle and ground reaction forces[J]. Frontiers in Bioengineering and Biotechnology, 2019, 7: 244.

[12] O'Connor J D, Rutherford M, Bennett D, et al. Long-term hip loading in unilateral total hip replacement patients is no different between limbs or compared to healthy controls at similar walking speeds[J]. Journal of biomechanics, 2018, 80: 8-15.

[13] Wu G, Caro S, Bai S, et al. Dynamic modeling and design optimization of a 3-DOF spherical parallel manipulator[J]. Robotics and Autonomous Systems, 2014, 62(10): 1377-1386.

[14] Wu G, Caro S, Wang J. Design and transmission analysis of an asymmetrical spherical parallel manipulator[J]. Mechanism and Machine Theory, 2015, 94: 119-131.

[15] F. Bidault, C.-P. Teng, J. Angeles, Structural optimization of a spherical parallel manipulator using a two-level approach, ASME Design Engineering Technical Conferences and Computers and Information in Engineering Conference, 2001, Pittsburgh, Pennsylvania, 2001. 